\title{
A simple micro-incinerator
}

\author{
Milton Thiago de Mello \\ Instituto Oswaldo Cruz, Rio de Janeiro, D.F.
}

(With one text-figure)

The increasing use of mineral oil for the preservation of cultures of bacteria and fungi (ARÊA-LEÃo \& CURY, 1950; HARTSELL, 1953) gave origin to a serious problem during the sterilization of the loops utilized for the transfer of such cultures. In fact, the mixture of mineral oil with the aqueous components of the media and the microorganisms, splatters suddenly when the loop is introduced into the open flame of a gas burner. The aerosols thus formed are heavier than those formed during the flaming of loops carrying only small quantities of common microorganisms (ANDERSON et al., 1950-1952) .

Due to the presence of fatty substances in Mycobacteria, the flaming of loops used with these microorganisms is also very dangerous, a fact which has always been recognized.

To avoid the formation of aerosols during the transfer of cultures preserved under mineral oil (usually in stabs in semi-solid media) Pasteur pippettes are generally employed, and discarded with disinfectant after use. This procedure, of course, is time-consuming and expensive when many cultures have to be transferred daily as is the case in large culture collections. Moreover it is not useful when the cultures are maintained in solid media.

The use of some sort of glass protectors for the burners, chiefly when working with Mycobacteria, does not prevent the dissemination of aerosols. Some recent modifications, however, the so-called micro- 
-incinerators, are safer, but they are not easily available in most laboratories.

In routine work with pathogenic microorganisms preserved under mineral oil, especially in the constant handling of Brucella and Mycobacterium species, we use a very simple micro-incinerator that can be mounted in any laboratory .

The device needs only a 18 or $20 \mathrm{~mm}$ x $200 \mathrm{~mm}$ test tube of fireresistant glass (for instance Vycor or Pyrex). It is fixed at an angle of about $45^{\circ} \mathrm{C}$ in a metallic support in front of the operator, in such a manner that a loop can be easily introduced into it.

During the operation the flame of a Bunsen burner is maintained under the middle part of the tube, without reaching any of its two extremities. With an ordinary Pyrex test tube $18 \times 150 \mathrm{~mm}$, when an open flame of $1250^{\circ} \mathrm{C}$ reaches its external wall, the inside temperature is about $800^{\circ} \mathrm{C}$, as measured with thermo-couple. When a $30 \times 200 \mathrm{~mm}$ test tube is used with the same flame, the temperature inside the tube is about $710^{\circ} \mathrm{C}$. In both cases a bit of cotton wool introduced slowly into the tube burns explosively when it reaches the super-heated zone.

The loop with the material, the splattering of which must be avoided, is introduced rapidly into the tube (taking care so as to not. touch the wall of the tube) until it reaches the botton. The portion of the loop with the microorganisms is then in the least heated zone of the tube, where the material is gradually dissecated (fig. 1). The minimum amounts of aerosols that may be accidentally formed are immediately burned when they pass through the super-heated atmosphere of the middle part of the tube.

After the apparent dissecation, the loop is slowly pulled out of the tube and maintained in the super-heated zone for some seconds and the wall of the tube may now be touched. the tube.

The final sterilization of the handle of the loop is made outside

\section{REFERENCES}

Anderson, R.A., Moss, M.L. \& Gross, N.H., 1952, Potential infectious hazards of common bacteriological techniques. Bact. Proc., Soc. Amer. Bact., 5th Gen. Meet., 58 .

Anterson, R.A., Stein, L., Moss, M.L. \& Gross, N.H., 1952, Potential infectious hazards of common bacteriological techniques. J. Bact., 64(4):473-481.

ARtê-LEÃo, A.E. \& CURY, A., 1950, Preservation of pathogenic fungous cultures under mineral oil. 5th International Congress for Microbiology, Rio de Janeiro. (In press).

HartSEll, S.E., 1953, The preservation of bacterial cultures under paraffin oil. Appl. Microb., 1:36-41. 